\title{
Analysis of LINE-I Elements in DNA from Postmortem Brains of Individuals with Schizophrenia
}

\author{
Glenn A Doyle*,', Richard C Crist', Emre T Karatas', Matthew J Hammond', Adam D Ewing', \\ Thomas N Ferraro ${ }^{3}$, Chang-Gyu Hahn' and Wade H Berrettini' \\ 'Translational Research Laboratories, Center for Neurobiology and Behavior, Department of Psychiatry, University of Pennsylvania Perelman School \\ of Medicine, Philadelphia, PA, USA; ${ }^{2}$ Mater Research Institute_-University of Queensland, Brisbane, Australia; ${ }^{3}$ Department of Biomedical Sciences, \\ Cooper Medical School of Rowan University, Camden, NJ, USA
}

\begin{abstract}
Whereas some rare genetic variants convey high risk for schizophrenia (SZ), common alleles conveying even moderate risk remain elusive. Long interspersed element- IS (LI) are mobile retrotransposons comprising I7\% of the human genome. LI retrotransposition can cause somatic mosaicism during neurodevelopment by insertional mutagenesis. We hypothesized that, compared to controls, patients diagnosed with schizophrenia (PDS) may have increased numbers of deleterious LI insertions, perhaps occurring de novo, in brain-expressed genes of dorsolateral prefrontal cortex (DLPFC) neurons. Neuronal and non-neuronal nuclei were separated by fluorescence-activated cell sorting from postmortem DLPFC of 36 PDS and 26 age-matched controls. Genomic sequences flanking the 3'-side of LIs were amplified from neuronal DNA, and neuronal LI libraries were sequenced. Aligned sequences were analyzed for $L I$ insertions using custom bioinformatics programs. Ontology and pathway analyses were done on lists of genes putatively disrupted by LIs in PDS and controls. Cellular or population allele frequencies of LIs were assessed by droplet digital PCR or Taqman genotyping. We observed a statistically significant increase in the proportion of intragenic novel LIs in DLPFC of PDS. We found over-representation of LI insertions within the gene ontologies 'cell projection' and 'postsynaptic membrane' in the gene lists derived from PDS samples, but not from controls. Cellular allele frequencies of examined LI insertions indicated heterozygosity in genomes of DLPFC cells. An LI within ERII exoribonuclease family member 3 (ER/3) was found to associate with SZ. These results extend prior work documenting increased LI genetic burden in the brains of PDS and also identify unique genes that may provide new insight into the pathophysiology of schizophrenia.

Neuropsychopharmacology (2017) 42, 2602-26II; doi:I0.1038/npp.2017.II5; published online 5 July 2017
\end{abstract}

\section{INTRODUCTION}

Schizophrenia (SZ) is a devastating chronic psychotic illness affecting $0.5-1.0 \%$ of the population worldwide. The inherited component to risk for SZ is estimated at $64 \%$ (Lichtenstein et al, 2009). Genome-wide association studies (GWAS) with sample sizes exceeding 50000 persons reported more than 100 genetic loci containing common alleles conveying only minor influences (odds ratios $<1.2$ ) on risk (Ripke et al, 2014). Rare copy number variations increase disease risk substantially, but only in a small fraction of patients diagnosed with SZ (PDS) (Lee et al, 2012 for review).

Somatic brain DNA variations have been found to contribute to the incidence of early-onset Alzheimer's disease (Beck et al, 2004), brain malformations (Evrony et al, 2012), and Sturge-Weber syndrome (Shirley et al, 2013; Nakashima

*Correspondence: Dr GA Doyle, Translational Research Laboratories, Center for Neurobiology and Behavior, Department of Psychiatry, University of Pennsylvania Perelman School of Medicine, Room 2210, I 25 South 31 st Street, Philadelphia, PA 19104, USA, Tel: + I-215-5734583, Fax: + I-215-573-204I, E-mail: gadoyle@mail.med.upenn.edu Received 9 March 2017; revised 10 May 20 I7; accepted 31 May 2017; accepted article preview online 6 June 2017 et al, 2014). Thus, one novel approach to identifying SZ risk alleles is the study of somatic mutations within brain DNA sequence.

One source of somatic brain DNA variation is long interspersed element-1 (L1) retrotransposons (Muotri et al, 2005, 2010; Coufal et al, 2009). Full-length L1s are $\sim 6 \mathrm{~kb}$ mobile DNA elements with a promoter, 5'-UTR, 3'-UTR, and two open reading frames encoding an RNA-binding protein and an endonuclease/reverse transcriptase fusion protein (Mathias et al, 1991; Feng et al, 1996). L1s multiply and accumulate in the host genome by a 'copy-and-paste' mechanism; they are estimated to comprise $\sim 17 \%$ of the human genome (Lander et al, 2001; Beck et al, 2010). Most L1s have lost their 'genomic mobility' due to truncations or mutations (Ostertag and Kazazian, 2001), but 100 fulllength 'competent' L1s, mostly from the human-specific L1 (L1Hs) Ta subfamily, are capable of retrotransposition in the human genome (Beck et al, 2010; Brouha et al, 2003).

Kazazian et al (1988) first demonstrated that a germ line L1 retrotransposition event caused human disease. Since then, $>125$ cases of germ line L1-mediated gene disruption have been reported to cause Mendelian diseases (Goodier, 2014; Hancks and Kazazian, 2016). Complex diseases such as neurofibromatosis type I (Vogt et al, 2007), chronic 
granulomatous disease (Roos et al, 2010), primary immunodeficiency (de Boer et al, 2014), colon cancer, and choroideremia (Hancks and Kazazian, 2016) have been caused by somatic L1 retrotransposition into various, different genetic loci. These somatic L1-mediated diseases provide a rationale for the hypothesis that brain somatic L1 retrotranspositions may contribute to psychiatric disease risk.

Bundo et al (2014) studied L1 burden in gDNA from dorsolateral prefrontal cortex (DLPFC) and liver of three PDS and three control individuals by whole-genome sequencing. Gene Ontology (GO) analysis yielded significant over-representation of SZ brain-specific L1s in genes for the GO terms 'synapse' (Bonferroni corrected $p=3 \times 10^{-9}$ ), 'synapse part' $\left(p=4.4 \times 10^{-5}\right)$, and 'plasma membrane part' $\left(p=1.5 \times 10^{-5}\right)$. Many of the genes harboring L1s have been implicated previously in GWAS of PDS (Bundo et al, 2014; Ripke et al, 2014).

We studied postmortem DLPFC from PDS and normal controls. DLPFC tissue was studied because PDS have working memory deficits and semantic and verbal memory loss, dysfunctions involving DLPFC (Gur et al, 2001; Carter et al, 2009). Brain imaging has revealed abnormal DLPFC activity among PDS (Weinberger et al, 1986; Manoach et al, 2000; Carter et al, 1998; Curtis et al, 1999). This study, done using different methodology, serves as a confirmation and extension of the results published by Bundo et al (2014).

\section{MATERIALS AND METHODS}

\section{Study Subjects}

All postmortem brain samples were obtained from the University of Pennsylvania BioBank brain collection. Supplementary File S1 contains data on brain tissue characteristics, including postmortem interval (PMI), weight, RNA integrity number (RIN), and $\mathrm{pH}$, as well as complete subject demographics, including age at onset and chlorpromazine equivalents for the 36 PDS (mean age $=79 \pm 10 ; 64 \%$ female; 34 European-American and 2 African-American) and 26 controls (mean age $=67 \pm 14 ; 54 \%$ female; 18 European-American, 7 African-American, and 1 multiracial) studied. Before death, the diagnosis of SZ was established for all patients after examination by a psychiatrist. All subjects had at least one full antemortem clinical assessment, as described (Arnold et al, 1995). Assessments are DSM-IV diagnosis, Brief Psychiatric Rating Scale, history of medications, Scale for Assessment of Negative Symptoms, and Scale for Assessment of Positive Symptoms. Control individuals had no history of psychiatric illness and had a nonneurological cause of death.

\section{Blood DNA Samples from PDS and Controls}

De-identified gDNA from EBV-transformed lymphoblastoid cell lines of European-American subjects who met DSM-IV criteria for SZ $(n=1316)$ and controls $(n=2303)$ were acquired from the Rutgers University Cell and DNA Repository (Infinite Biologics, Piscataway, NJ, USA). gDNA was diluted to $20 \mathrm{ng} / \mu \mathrm{l}$ in sterile water before use in genotyping experiments.

\section{Neuronal Nuclei Isolation}

L1s mobilize more frequently during differentiation of neurons than during differentiation of non-neuronal cell types (Coufal et al, 2009), and L1s can mobilize in postmitotic neurons (Macia et al, 2017). Thus, we chose to enrich for and analyze neuronal cell genomes for L1 retrotransposition. We isolated neuronal and non-neuronal nuclei from frozen postmortem DLPFC tissue (Jiang et al, 2008). A unit of 10-15 mg (wet weight) of individual DLPFC samples were used to create seven pools for analysis: four pools were derived from 8-10 individual DLPFC samples from PDS and three pools were derived from DLPFC samples from 8-10 individual controls (Supplementary File S1). Each pool of DLPFC samples was thawed and lysed simultaneously by homogenization in ice-cold $0.32 \mathrm{M}$ sucrose solution containing $0.5 \%$ Triton X-100, lysates layered onto a $1.8 \mathrm{M}$ sucrose cushion, and nuclei pelleted by ultracentrifugation $\left(\sim 107000 \mathrm{~g}, 2.5 \mathrm{~h}, 4{ }^{\circ} \mathrm{C}\right)$. Pelleted nuclei were suspended in $1 \times$ phosphate-buffered saline containing $3 \mathrm{mM} \mathrm{MgCl}_{2}$, labeled with an AlexaFluor-488-conjugated anti-NeuN antibody (Millipore, Temecula, CA, USA), counterstained with diamidino-2-phenylindole (Thermo-Fisher Scientific, Waltham, MA, USA) and strained through a $30 \mu \mathrm{m}$ filter-cap tube (Becton-Dickinson, Franklin Lakes, NJ, USA). Labeled nuclei were sorted into NeuN-positive (neuronal) and NeuNnegative (non-neuronal) populations on an AriaII fluorescence-activated cell sorter (Beckman-Coulter, Brea, CA, USA). Sorted nuclei were pelleted by centrifugation $\left(2000 \mathrm{~g}, 30 \mathrm{~min}, 4^{\circ} \mathrm{C}\right)$, then lysed in $1 \times$ proteinase $\mathrm{K}$ digestion buffer, incubated with $0.857 \mu \mathrm{g} / \mu \mathrm{l}$ proteinase $\mathrm{K}$ at $56^{\circ} \mathrm{C}$ for $16 \mathrm{~h}$, and gDNA purified by chloroform extraction and ethanol precipitation in the presence of $20 \mu \mathrm{g}$ molecular biology grade glycogen (Roche, Indianapolis, IN, USA). All DNA samples were quantified by fluorimetry using the Quant-it high-sensitivity double-stranded DNA kit (Thermo-Fisher Scientific). Although not all gDNA samples were assessed for intact high-molecular-weight DNA by gel electrophoresis, post-FACS-sorted gDNA samples prepared in our lab by these methods showed the purified gDNA migrated above the $23 \mathrm{kbp}$ fragment of the HindIII-digested lambda DNA size marker without evidence of excessive degradation.

\section{L1 Amplification and Sequencing}

Purified gDNA from NeuN-positive nuclei was subjected to L1-seq as described (Ewing and Kazazian, 2010), except primary PCR was done using $25 \mathrm{ng}$ of gDNA template for each of the eight hemi-specific degenerate primer reactions. DNA amplicons of each degenerate primer reaction were size selected by gel electrophoresis and purified. DNA concentration of the L1 amplicons from each reaction was assessed by Agilent high-sensitivity double-stranded DNA chip on an Agilent BioAnalyzer 2100. L1 amplicons were then mixed in equimolar quantities to produce the amplicon libraries for sequencing. Final library concentrations were assessed by KAPA Biosystems Illumina qPCR before clustering on the Illumina HiSeq2000. Amplicon libraries were sequenced using 100 nucleotide single-end reads in one lane (per pool library) of an Illumina HiSeq2000. 


\section{L1-seq Bioinformatics}

L1-seq bioinformatics analysis was done using the most current version (available at https:/github.com/adamewing/ 11seq) of the L1-seq software (Ewing and Kazazian, 2010). Briefly, sequencing reads were trimmed to remove 5'-primer sequence and 3'-low-quality bases, and aligned to human genome build hg19 using bowtie2-2.1.0 (Langmead and Salzberg, 2012). Samtools-0.1.19 (Li et al, 2009) converted aligned reads to bam files. Peaks consisting of aligned reads within a common $500 \mathrm{bp}$ window were identified and used to calculate putative L1 insertion points. Predicted L1 insertions were compared across sample populations and annotated as being reference (a genetically 'fixed' L1 in the human reference (hg19) genome), known non-reference (KNR; a previously described 'polymorphic' L1 in the human population, but not in the reference genome), or previously uncataloged (novel; a previously 'unidentified' L1 in the human population) L1s by comparison of L1 positions to a catalog of published L1 insertion data sets (Ewing and Kazazian, 2010; Sudmant et al, 2015). Identified novel and KNR L1 insertions were screened for high quality in Excel (Microsoft, Redmond, WA, USA). Quality metrics for an L1 insertion were that the reads aligned well to the reference genome ('mean mapq' $\geqslant 30$, 'mappability'>0.5), had $>98 \%$ average match ('mean matchpct' $>0.98$ ), at least six total reads across pooled populations ('total reads' $>5$ ), and at least two unique reads in a 'peak' ('unique alignments' $>1$ ). All subsequent analyses were limited to intragenic L1 insertions, defined as a predicted L1 located within an intron, an exon, or within 500 bp of a transcription start site or 3'-UTR.

\section{LINE-1 Validations}

Supplementary File S2 contains information on L1 validations attempted (successful validations are highlighted in green). Owing to the paucity of neuronal NeuN-positive gDNA remaining after L1-seq library constructions, wholegenome amplification (WGA) by multiple displacement amplification was performed on $10 \mathrm{ng}$ of each NeuNpositive and NeuN-negative gDNA sample using a Repli-G mini kit (Qiagen, Hilden, Germany) before PCR validation with GoTaq polymerase (Promega, Madison, WI, USA), $10 \mathrm{ng}$ WGA DNA, and $0.2 \mu \mathrm{M}$ primers in a $25 \mu \mathrm{l}$ reaction. We used the Primer3 program (Rozen and Skaletsky, 2000) to design genome-specific 'filled site' (FS) and 'empty site' (ES) primers for confirmatory PCR (Ewing and Kazazian, 2010). Two initial primer pairings, FS with ES and FS with L1HsTAILSP1AP2 (L1HsT, 5'-GGGAGATATACCTAATG CTAGATGACAC-3'), were used to amplify the genomic region surrounding or including, respectively, a putative L1 insertion (Ewing and Kazazian, 2010). When necessary, we performed nested PCR using $2 \mu \mathrm{l}$ of the initial PCR (FS-L1HsT primer pairing) as template and nested 'FS' and L1-specific (L1HsG, 5'-TGCACATGTACCCTAAAACTTAG-3') primers. Gel-purified amplicons were cloned by ligation into pCRII-TOPO (Thermo-Fisher Scientific), ligation products transformed into bacteria, and purified plasmid DNA containing putative L1 insertions Sangersequenced using big-dye chemistry (Supplementary File S3). Resultant sequences were queried against hg19 using BLAT

\footnotetext{
Neuropsychopharmacology

(Kent, 2002). To determine the occurrence of confirmed L1 inserts among individuals of a pool, PCR was performed on bulk gDNA purified from a second DLPFC sample isolated from each individual in the pool.

\section{Taqman Genotyping of $\mathrm{L} 1$ in $E R I 3$}

We developed an allelic discrimination Taqman assay for detection of the ERI1 exoribonuclease family member 3 (ERI3) alleles with and without the validated L1. Briefly, two probes that overlapped the L1 insertion point were used: a FAM-labeled probe was designed to detect the L1-containing allele; and a VIC-labeled probe was designed to detect the normal, L1-lacking allele. Three primers were used: two genome-specific primers (forward and reverse) surrounding the ERI3-L1 insertion point enabled detection of the L1-lacking allele; and the third primer (L1HsG) allowed detection of the L1-containing allele. Cycling parameters were denaturation at $95^{\circ} \mathrm{C}$ for $10 \mathrm{~min}$ followed by 40 cycles of $95^{\circ} \mathrm{C}, 15 \mathrm{~s} ; 56^{\circ} \mathrm{C}, 5 \mathrm{~s}$; and $60^{\circ} \mathrm{C}, 1 \mathrm{~min}$. Reaction volumes were $5 \mu \mathrm{l}$ containing $40 \mathrm{ng}$ gDNA as template. Amplified PCR reactions were post-read on an ABI 7900HT with two-cluster calling enabled.

\section{Droplet Digital PCR: Cellular Allele Frequency Determinations}

L1Hs- and gene-specific primers designed to amplify 200-250 bp products were used for droplet digital PCR (ddPCR). The L1Hs-specific 5'-FAM-labeled probe was designed as described (White et al, 2014) except it was double-quenched with 3'-Iowa Black and internal ZEN quenchers (Integrated DNA Technologies). Approximately $100 \mathrm{ng}$ of gDNA was XmnI-digested in ddPCR master mix at $37^{\circ} \mathrm{C}$ for $1 \mathrm{~h}$ before droplet formation using a QX100 droplet generator (Bio-Rad, Hercules, CA, USA). Droplets were cycled at $95^{\circ} \mathrm{C}$ for $10 \mathrm{~min}, 40$ cycles of $94^{\circ} \mathrm{C}, 30 \mathrm{~s} ; 60^{\circ} \mathrm{C}$, $1 \mathrm{~min}$; then $98^{\circ} \mathrm{C}, 10 \mathrm{~min}$; and $12^{\circ} \mathrm{C}$ soak. Amplified droplets were read using a QX200 droplet reader (Bio-Rad) under absolute quantification for FAM (L1 allele probe, unknown) or VIC (RPPH1 allele probe, reference, ThermoFisher Scientific; Catalog No. 4403328) fluorescence. QuantaSoft v1.7.4 (Bio-Rad) was used for data analysis and graph generation.

\section{Database for Annotation, Visualization, and Integrated Discovery Analyses}

L1-seq bioinformatics analysis generated lists of genomic L1 positions within the PDS and control populations. The Database for Annotation, Visualization, and Integrated Discovery (DAVID; version 6.7 or 6.8; Huang et al, 2009) algorithm was used to assess lists of genes harboring either novel or KNR L1 insertions for enrichment of GOs and for Kyoto Encyclopedia of Genes and Genomes (KEGG) pathways.

Analyses were done with DAVID 6.7 because this is the version that was used in Bundo et al (2014), thus allowing direct comparison of our results with theirs. DAVID 6.8 analyses were also done (Supplementary Table S1 in Supplementary Information) because this version contains the most current Knowledgebase of GOs and pathways. In 
each case, default settings were used. Supplementary File S4 contains information on the analyzed gene lists corresponding to intragenic L1s found in the control or PDS populations (Supplementary File S5).

\section{Statistics}

DAVID uses a hyper-geometric distribution to calculate fold enrichment and $p$-values (Huang et al, 2009; see Methods in Supplementary Information). 'Raw' $p$-values calculated by DAVID were adjusted by Bonferroni correction for multiple testing. Goodness-of-fit (Kolmogorov-Smirnov) test for normal distribution of data was done online at http:// contchart.com/goodness-of-fit.aspx. To assess the length of genes harboring novel L1 insertions, an unpaired $t$-test assuming equal variance was done on the normally distributed $\log _{10}$-transformed data using GraphPad Prism version 4.00 for Windows (GraphPad Software, San Diego, CA, USA). All other statistical analyses comparing the number of L1 insertions within and among populations were done by $\chi^{2}$-tests online at https://www.medcalc.org/calc/ comparison_of_proportions.php or at http://www.quantpsy. org. All reported $p$-values are two-tailed.

\section{RESULTS}

The numbers of total and intragenic KNR and novel L1 insertions, meeting our criteria, are given in Table 1 (also Supplementary Figure S1 in Supplementary Information). Given previous estimates using a linear regression model for the number of non-reference L1Hs elements segregating in populations of various sizes (Ewing and Kazazian, 2010), we expected 863 and 901 total non-reference L1 insertions in our control (26 individuals) and PDS (36 individuals) populations, respectively. We observed 903 and 937 total L1 insertions in our control and PDS populations, respectively; these values were not significantly different from expectations based on counts $\left(\chi^{2}=0.008, p=0.929\right)$. Comparisons of the numbers of intragenic to intergenic L1 insertions among populations indicated a statistically significant increase in the proportion of intragenic novel L1 insertions in DLPFC of PDS; the PDS population had $\sim 15 \%$ more populationspecific intragenic novel L1s than controls (Table 1). In contrast, the proportion of intragenic KNR L1 insertions was not statistically different (Table 1). Thus, only the intragenic novel L1 insertions were increased in the SZ cases studied here. There was no difference between the control and PDS group with regard to the lengths of genes in which novel L1 insertions were detected (unpaired $t$-test; $p=0.851$ ).

Our PCR confirmation rate for intragenic novel L1 insertions identified in the PDS cohort was $~ 50 \%$ (Supplementary File S2). Some validations were attempted on L1s that did not meet our final quality criteria. Removal of these L1s from the validation list (four unsuccessful) increased our effective confirmation rate to $\sim 64 \%$. L1 insertions in ERI3, GRID2, KHDRBS2 (Figure 1a-c), NRG3, HTR2C, RYR2, SYNE1, SYN3, and ABCF1 (data not shown; Supplementary File S3) were confirmed by conventional PCR. ddPCR data (Figure 2) suggest that the GRID2, KHDRBS2, and SYNE1 L1s are germ line or occurred de novo very early during development in the affected individuals because they had L1:RPPH1 ratios of $\sim 50 \%$. The GRID2 L1 insertion may be a personal de novo mutation because it was detected only in the DLPFC gDNA of 1 of 33 PDS, and it was not observed in lymphoblastoid gDNA from 995 SZ cases by Taqman assay (data not shown). The ERI3 L1 insertion is germ line and was significantly more common $\left(\chi^{2}=4.323, p=0.0376\right)$ in lymphoblastoid gDNA from SZ cases of European origin (minor allele frequency $=1.9 \%$, $n=1316$ ) compared to controls (minor allele frequency $=$ $1.3 \%, n=2303)$.

Bundo et al (2014) reported 751 and 385 genes with brainspecific L1 insertions in their SZ and control populations, respectively. In comparing our PDS vs control cohorts, we identified 230 vs 249, and 194 vs 143 non-redundant genes harboring KNR and novel L1s, respectively (Supplementary File S4). A total of 36 vs 29 genes containing KNR L1s in our SZ and control cohorts, respectively, were among those genes identified with brain-specific L1 insertions in the PDS and the control populations of Bundo et al (2014; Table 2A and C). In all, 27 vs 7 genes containing novel L1 insertions in our PDS and control cohorts, respectively, were among those genes identified with brain-specific L1s in the SZ and control populations of Bundo et al (2014) (Table 2B and D). At least 15 of 36 genes containing KNR L1 insertions in the PDS cohorts of this study and Bundo et al (2014) have a genetic association with SZ, including disruption by CNVs (Lee et al, 2012; Table 2A). Similarly, at least 8 of the 27 genes containing novel L1 insertions identified in both PDS cohorts have an association with SZ (Table 2B). In contrast, at least 10 of the 29 and 1 of 7 genes containing KNR and novel L1 insertions, respectively, in the normal cohorts have

Table I Statistical Analyses of LI Proportions (Intragenic vs Intergenic) Within Each Population

\begin{tabular}{|c|c|c|c|c|c|}
\hline LI category & Nm intra/total (\% intra) & SZ intra/total (\% intra) & $\chi^{2}$ & $p$-value* & Difference (SZ-Nm) \\
\hline Total novel LIs & $122 / 259(47.1)$ & I 66/307 (54.07) & 2.726 & 0.0987 & $6.97 \%$ \\
\hline Population-specific novel LIs & $44 / 98(44.90)$ & $88 / 146(60.27)$ & 5.556 & 0.0184 & $15.37 \%$ \\
\hline Total KNR LIs & $241 / 644(37.42)$ & $231 / 630(36.67)$ & 0.077 & 0.7818 & $0.75 \%$ \\
\hline Population-specific KNR LIs & $50 / 126(39.68)$ & $40 / 112(35.7 \mid)$ & 0.396 & 0.5293 & $3.97 \%$ \\
\hline Total novel+KNR LIs & $363 / 903(40.2)$ & $397 / 937(42.4)$ & 0.917 & 0.338 & $2.20 \%$ \\
\hline Population-specific novel+KNR LIs & $94 / 224(42.0)$ & $128 / 258$ (49.6) & 2.811 & 0.0936 & $7.60 \%$ \\
\hline
\end{tabular}

Abbreviations: KNR, known non-reference; Nm, normal cohort, Sz, schizophrenia cohort.

* $p$-values in bold are statistically significant, defined as $p<0.05$. 


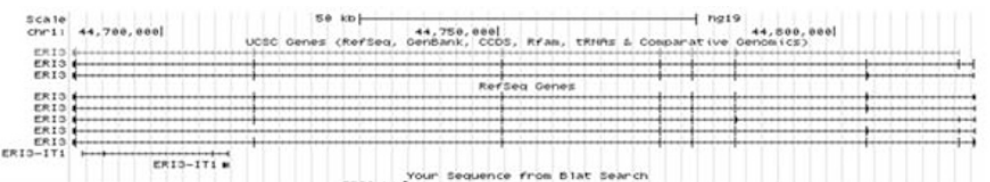

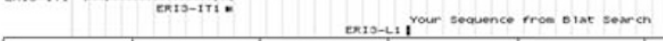
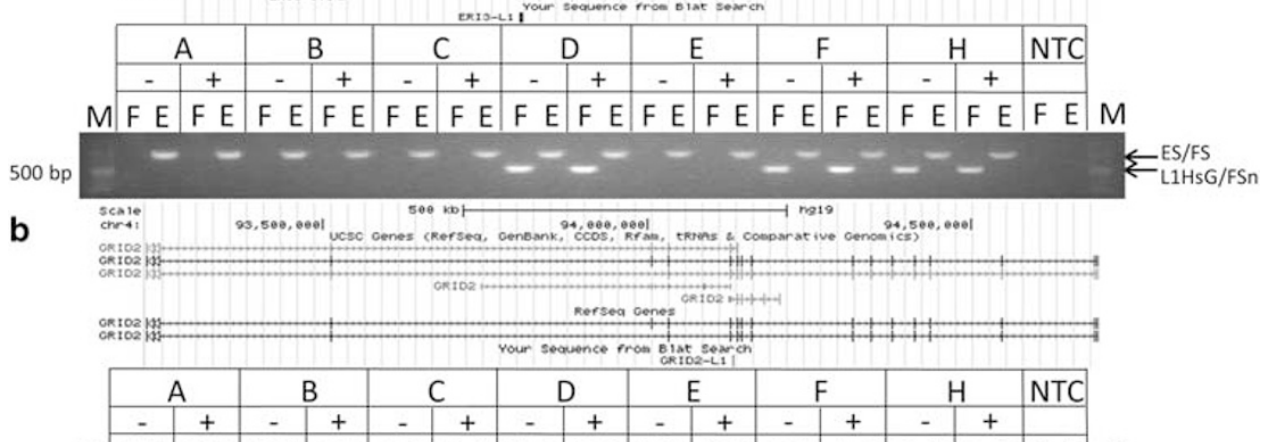

M) FE FE FE FE FE FE FE FE FE FE FE FE FE FE FE M

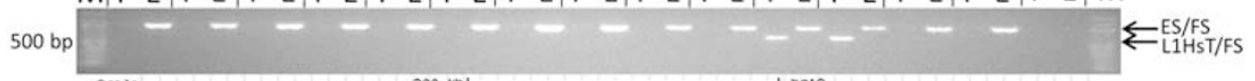

C

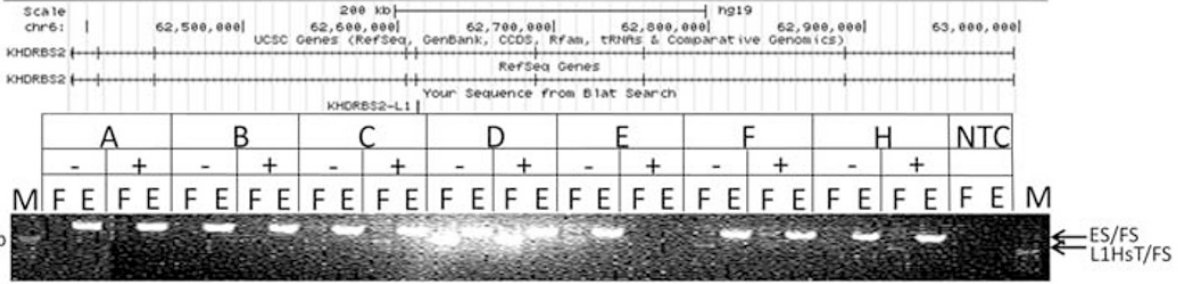

Figure I PCR confirmation of novel LI RTP insertions in various genes. Gel images showing the 'filled site' ('F'; LIHsT/FS or LIHsG/FS primer pair) and 'empty site' ('E'; ES/FS primer pair) PCR amplicons. The LI insertions were detected in both the NeuN-negative (-) and NeuN-positive (+) gDNA reactions. (a) ERII exoribonuclease family member 3 (ER/3). (b) Glutamate receptor, ionotropic, delta 2 (GRID2). (c) KH RNA-binding domain-containing, signal transduction-associated 2 (KHDRBS2). All LIs were confirmed in the pools in which each predicted LI insert was initially detected. Lane 'M' is the NEB I00 bP marker. Lanes A, C, and E are normal control populations; lanes B, D, F, and $\mathrm{H}$ are SZ populations. Minus or plus signs indicate amplicons of gDNA from NeuN-negative (not sequenced, non-neuronal) or NeuN-positive (sequenced, neuronal) nuclei, respectively.

an association with SZ (Table 2C and D). Notably, of 210 novel, intragenic L1 insertions identified in our analysis, only one (in an intron of NPAS1) was potentially identical by position (chr19:47540689 vs chr19:47542090) to a brainspecific L1Hs identified by Bundo et al (2014).

Several genes identified as having L1 insertions in the PDS cohorts of Bundo et al (2014) and the present study were common among the 350 genes within the 108 loci identified by the SZ GWAS (Ripke et al, 2014; Supplementary Figure S2 in Supplementary Information). The number of genes found in common with the SZ GWAS was not statistically different between our study and Bundo et al (2014) for the PDS $\left(\chi^{2}=0.126, p=0.723\right.$ for KNR; $\chi^{2}=0.149, p=0.699$ for novel) or control $\left(\chi^{2}=0.003, p=0.956\right.$ for KNR; $\chi^{2}=0.092$, $p=0.762$ for novel) populations. Of $350 \mathrm{SZ}$ candidate genes identified by GWAS (Ripke et al, 2014), only CSMD1 was found in common among the L1-containing genes identified in the PDS populations of Bundo et al (2014) and the present study.

Significant GO terms were determined by DAVID 6.7 analyses (Table 3). For novel intragenic L1s, the GO term 'cell projection' was only statistically significant in the PDS group, regardless of inclusion of genes in common between the two populations (Table $3 \mathrm{~A}$ and $\mathrm{B}$ ). Consistent with Bundo et al (2014), KNR intragenic L1 insertions found in our study showed statistical significance for the GO term 'plasma membrane part' only in the PDS group (Table 3C).
In contrast to Bundo et al (2014), the GO terms 'synapse' and 'cell junction' were found in both the normal and PDS populations (Table 3C). There were no significant GO terms identified when genes containing KNR L1 insertions overlapping the two populations were excluded from the analysis (Table 3D). However, when population-specific lists of KNR and novel L1-containing genes were combined, 'regulation of apoptosis' was statistically significant only in the control population, whereas 'synapse' was significant only in the PDS population (Table 3E). DAVID 6.7 failed to identify significant KEGG pathways for either normal or PDS groups. When gene lists were analyzed with DAVID 6.8, 'postsynaptic membrane' was only statistically significant in the PDS population (Supplementary Table S1 in Supplementary Information).

\section{DISCUSSION}

Our results further document a statistically significant difference in DLPFC neuron L1 distribution between PDS and controls. Similar to Bundo et al (2014), the present study revealed an increased L1 burden in DLPFC from PDS (Table 1; Supplementary Figure S1 in Supplementary Information). However, only differences in the relative numbers of intragenic to intergenic novel, but not KNR, L1 insertions were significantly greater between PDS and controls (Table 1). These findings suggest that mutagenesis 

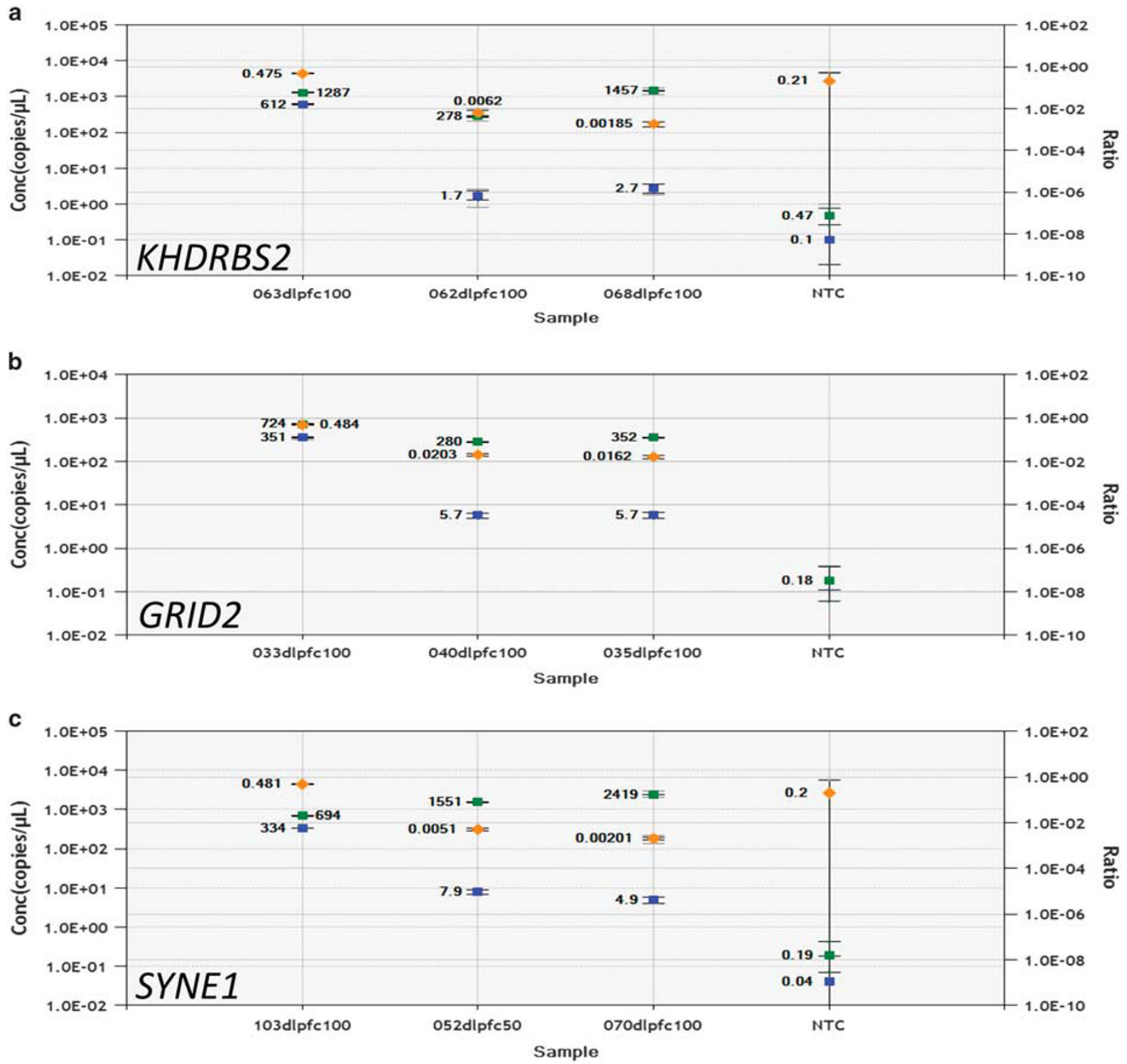

Figure 2 ddPCR allele frequency of LI s in various genes. Graphs show the absolute copy numbers of LI -containing genes (blue squares) and RPPHI (green squares) genes, as well as the ratios (Gene-LI :RPPHI, orange diamonds), detected in $100 \mathrm{ng}$ of Xmnl-digested gDNA from DLPFC various SZ samples. (a) The KHDRBS2-LI was detected in SZ patient 063, but not in SZ patients 062 or 068. (b) The GRID2-LI was detected in SZ patient 033, but not in SZ patients 035 or 040. (c) The SYNE I-LI was detected in SZ patient I03, but not in SZ patients 052 or 070. In all cases, the allele frequency of the LIs was $50 \%$, the theoretical frequency for an LI that is in heterozygosity in all genomes analyzed. NTC is the no template control.

by L1 retrotransposition during embryogenesis, or specifically during neurogenesis, may contribute to the etiology of SZ. Alternatively, the novel intragenic L1s may be rare hereditary insertions that disrupt gene function, leading to neurodevelopmental changes that increase risk for SZ.

One such example may be the L1 confirmed in ERI3 (Figure 1a). ERI3 interacts with the cellular prion protein during signal transduction (Spielhaupter and Schätzl, 2001), and other ERI family members are involved in the endogenous RNAi pathways of gene regulation (Thivierge et al, 2011; Duchaine et al, 2006). The pathophysiologic role of ERI3 in SZ is presently unclear; however, the fact that the relatively rare ERI3-L1 allele is polymorphic in the germ line, and was verified in multiple SZ individuals by PCR
(Figure 1a), and that Taqman genotyping assays (data not shown) showed it to be associated with $\mathrm{SZ}\left(\chi^{2}=4.323\right.$, $p=0.0376$ ) suggest $E R I 3$ disruption may increase risk for SZ.

Novel L1 insertions in GRID2 and KHDRBS2 were also confirmed (Figure $1 \mathrm{~b}$ and c). GRID2 expression is associated with pre-pulse inhibition of the acoustic startle response, an endophenotype for SZ (Swerdlow et al, 2013). A novel L1 in the serotonin $2 \mathrm{C}$ receptor gene HTR2C was also confirmed (data not shown; Supplementary File S3). Serotonin 2C receptors oppose the inhibitory effect of serotonin $1 \mathrm{~A}$ receptors on NMDA signaling in pyramidal neurons of prefrontal cortex (Yuen et al, 2008). Potential L1-mediated disruption of GRID2 and HTR2C expression in DLPFC may interfere with glutamate regulation, and, consistent with the 
Table 2 Genes Harboring LIs in Both Bundo et al (2014) and in the Present Study

\begin{tabular}{|c|c|c|c|}
\hline $\begin{array}{l}\text { (A) KNR LIs } \\
\text { (Sz) }\end{array}$ & $\begin{array}{l}\text { (B) Novel LIs } \\
\text { (Sz) }\end{array}$ & $\begin{array}{l}\text { (C) KNR LIs } \\
(\mathrm{Nm})\end{array}$ & $\begin{array}{l}\text { (D) Novel LIs } \\
\text { (Nm) }\end{array}$ \\
\hline ABLIM2 & AGBLI & ARHGAP24 & $B M P R / B$ \\
\hline ADAMTS $12^{\mathrm{a}}$ & ASTN2 $^{a}$ & C2orf86 & FCHSD2 \\
\hline BBS9 & $B M P R / B$ & $C A D M 2^{\mathrm{a}}$ & $P D E / A$ \\
\hline $\mathrm{Cl} 2$ orf55 & CLIP2 & CSMDI ${ }^{a}$ & PRHI \\
\hline C9orfl 71 & CREB5 & $D L G 2^{\mathrm{a}}$ & PTPRD \\
\hline$C A D M 2^{\mathrm{a}}$ & CSMDI $1^{\mathrm{a}}$ & $D M D$ & $U L K 4^{\mathrm{a}}$ \\
\hline CADPS $^{\mathrm{a}}$ & $\mathrm{DACH} 2$ & $D P P 6^{\mathrm{a}}$ & ZNF600 \\
\hline $\mathrm{CDH} / 3^{\mathrm{a}}$ & DIRC3 & $E R B B 4^{a}$ & \\
\hline $\mathrm{CDH} 4$ & DSCAM & FAM $19 A 2$ & \\
\hline CSMDI ${ }^{\mathrm{a}}$ & FAMI20C & FHIT & \\
\hline $\mathrm{DACH} 2$ & FAMI $55 \mathrm{~A}$ & GLIS3 & \\
\hline$D C C^{a}$ & $G P C 5^{\mathrm{a}}$ & JAK2 & \\
\hline$D L G 2^{a}$ & KSR2 & $K L H L 3$ & \\
\hline$E R B B 4^{\mathrm{a}}$ & $M A G / 2^{\mathrm{a}}$ & KLHL32 & \\
\hline FRMD4A & MARCHI & $\angle R P \mid B^{\mathrm{a}}$ & \\
\hline$G R I K 2^{a}$ & NCKAP5 & $N P A 3^{a}$ & \\
\hline$I L 2 \mid R$ & NPASI ${ }^{\text {a }}$ & OCA2 & \\
\hline KCNQ5 & $N R \times N 3^{\mathrm{a}}$ & PARD3B & \\
\hline KHDRBS2 & NSMCE2 & PRHI & \\
\hline KLHL3 & $P C D H / 5^{a}$ & PTPRD & \\
\hline KSR2 & PDZD2 & PTPRT & \\
\hline$\angle R P \mid B^{\mathrm{a}}$ & PTPRD & RAD5 ILI & \\
\hline MARCHI & RGLI & RALYL & \\
\hline MIPOLI & RPS6KCI & $R O B O I^{a}$ & \\
\hline ODZI & $T B C|D|$ & RYR3 $^{\mathrm{a}}$ & \\
\hline$O P C M L^{a}$ & UBE2H & SBF2 & \\
\hline PLD5 & $U L K 4^{a}$ & $S G C D$ & \\
\hline PTPRD & & SLC9A9 & \\
\hline RIMS2 $2^{\mathrm{a}}$ & & $U L K 4^{\mathrm{a}}$ & \\
\hline \multicolumn{4}{|l|}{ RORA } \\
\hline \multicolumn{4}{|l|}{$R Y R 3^{\mathrm{a}}$} \\
\hline \multicolumn{4}{|l|}{$S G C D$} \\
\hline \multicolumn{4}{|l|}{ SLC9A9 } \\
\hline \multicolumn{4}{|l|}{ SYNEI ${ }^{\mathrm{a}}$} \\
\hline \multicolumn{4}{|l|}{ UBE2H } \\
\hline$U L K 4^{\mathrm{a}}$ & & & \\
\hline
\end{tabular}

Abbreviations: Nm, normal cohort; Sz, schizophrenia cohort.

${ }^{a}$ Genes implicated in genetics of schizophrenia.

glutamatergic dysregulation hypothesis of SZ, increase risk for SZ.

ddPCR data (Figure 2) suggest the KHDRBS2, GRID2, and SYNE1 L1 insertions are possibly polymorphic in the germ line. This is likely true of the KHDRBS2 L1 because, unlike the GRID2 L1, it was found within the 1000 Genomes Project populations (Sudmant et al, 2015). KHDRBS2 regulates alternative splicing of neurexins (Iijima et al, 2014) that are themselves implicated in SZ pathophysiology (Brown et al, 2011). Although we cannot exclude the possibility that the GRID2 L1 is an extremely rare polymorphic L1 in the human population, allelic discrimination experiments (data not shown) suggest it could be a private, early developmental, somatic mutation in the PDS in whom it was identified. Inheritance of either putatively L1-disrupted allele could impact normal brain development through improper neurexin splicing (KHDRBS2-L1) or altered glutamatergic physiology (GRID2-L1). The confirmed L1 insertion in SYNE1 (Figure 2c) is likely polymorphic in the germ line because it was a KNR L1 (Table 2A). SYNE1 is a disrupted in SZ 1 (DISC1)-interacting protein (Costas et al, 2013) that has been implicated in bipolar disorder and other neuropsychiatric disorders (Xu et al, 2014). Indeed, SYNE1 was among 15 genes that showed overlapping associations with at least 5 of 6 neuropsychiatric disorders, including SZ (Lotan et al, 2014).

Several differences exist between our DAVID 6.7 GO results (Table 3) and those of Bundo et al (2014). Whereas some statistically significant GO terms were SZ-specific in both studies, some of the SZ-specific GO terms from Bundo et al (2014) were enriched in our normal cohort, for example, 'synapse' and 'cell junction' (Table 3C). The 'synapse' GO term was statistically significant in the controls of Bundo et al (2014) under their 'less stringent' criteria. In our analysis, SZ-specific GO terms included 'cell projection' (Table 3A and B), 'plasma membrane part', and 'plasma membrane' (Table 3C), as well as 'synapse' (Table 3E). Results of DAVID 6.8 analysis revealed 'postsynaptic membrane' as a SZ-specific GO term (Supplementary Table S1 in Supplementary Information). Given the different methods used (whole-genome vs L1 amplicon sequencing) and differences in sample populations, it is notable that our DAVID 6.7 and 6.8 GO term analyses are strikingly consistent with Bundo et al (2014). If it can be confirmed that the L1 insertions in genes within these ontologies disrupt gene function, it would bolster the theory that risk for SZ may partially reside in improper cell migration and junctions, leading to anatomically aberrant synaptic structures.

Several genes within the 'synapse' GO term have been implicated in SZ genetics and/or pathophysiology. Whereas most genes harboring L1 insertions were found in both our normal and PDS populations, 3 of 16 genes within the 'synapse' term (Table 3C) were specific to PDS: GABRA4; RIMS2; and SYNE1 (see above). Potential L1-mediated disruption of GABRA4 would be consistent with GABA receptor composition playing a role in SZ (Verdurand et al, 2013).

A recent meta-analysis of GWAS data sets yielded 108 genomic loci containing 350 genes (excluding the MHC locus genes) that were associated with SZ (Ripke et al, 2014). Notably, 11/350 SZ GWAS-implicated genes (ASPHD1, CNTN4, CSMD1, DPYD, GATAD2A, IMMP2L, KCTD13, KLC1, NEK1, PITPNM2, and TBC1D5) contained L1 insertions in our PDS population; 2/11 (ASPHD1 and KCTD13) were absent from our control population (Supplementary Figure S2 in Supplementary Information). Most were confirmed by detection in previous L1 studies (Supplementary File S5); however, L1 insertions within GATAD2A, KLC1, and PITPNM2 were novel, as was an L1 that 'hit' both ASPHD1 and KCTD13. Whether these L1 insertions disrupt gene expression or mark alleles with detrimental rare variants remains unknown.

Limitations to this study include the following: (1) limited power to detect SZ-associated L1 insertions due to the relatively small sample size; (2) detection of L1 insertions 
Table 3 DAVID 6.7 Analysis

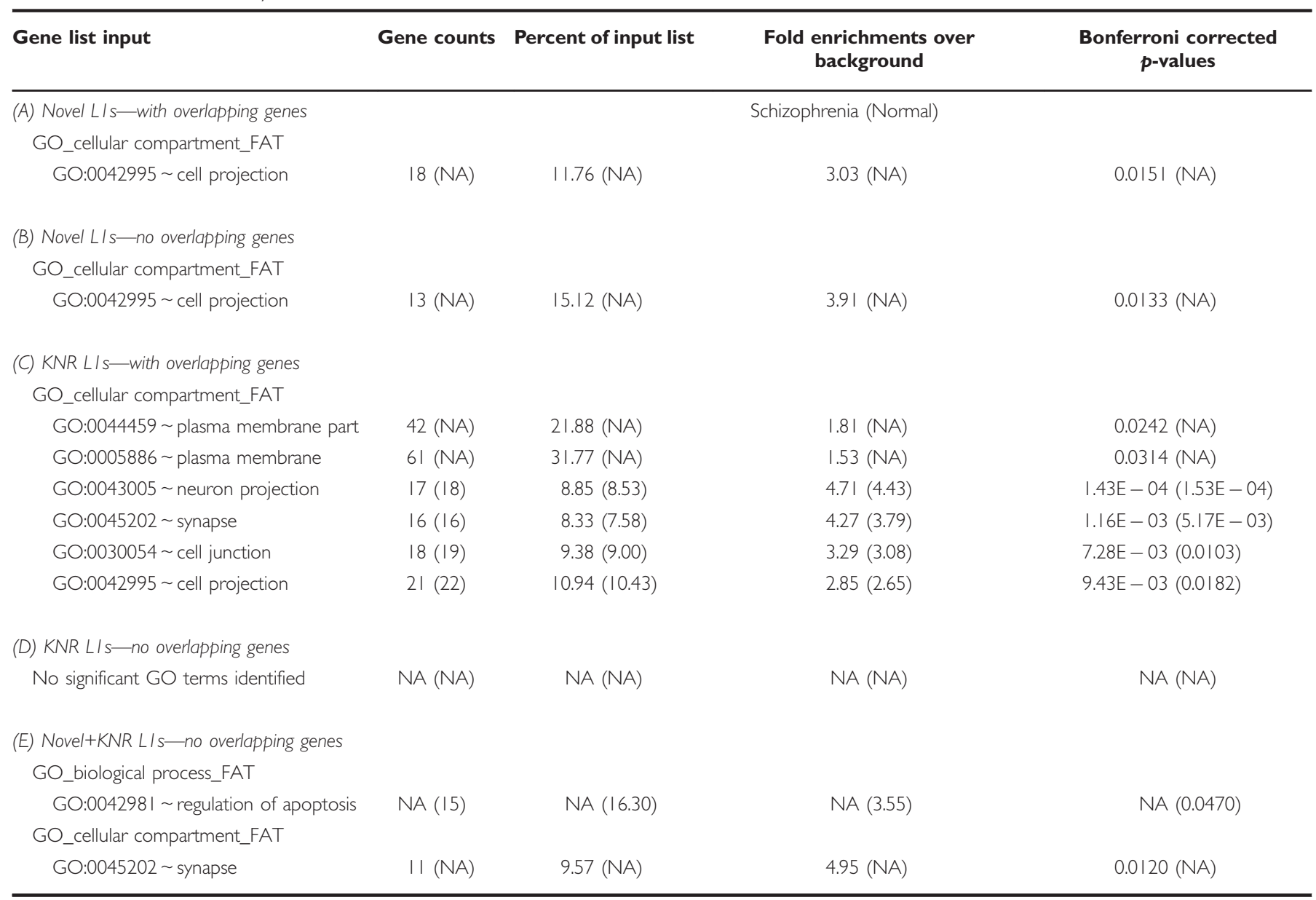

Abbreviations: FAT, filtered annotated terms; GO, Gene Ontology; KNR, known non-reference; NA, not applicable.

'Overlapping genes' refers to genes harboring LIs found in both the normal and schizophrenia populations.

limited to DLPFC given that other brain regions may be involved in SZ; (3) the population allele frequencies of most detected L1 insertions remain unknown making it difficult to firmly establish associations of each with SZ; (4) the functional significance of the intragenic L1 insertions remains unknown making mechanistic predictions related to SZ phenotypes currently speculative; (5) we analyzed only intragenic L1 insertions, but intergenic L1s could disrupt nearby gene function; (6) although no increase in relative genomic L1 content was observed when various antipsychotic medications were assayed in vitro (Bundo et al, 2014), we cannot exclude the possibility that certain medications taken by PDS in our cohort caused L1 mobilization in the DLPFC; and (7) the L1 insertions identified and confirmed in our study were found in both the glial $(\mathrm{NeuN}-)$ and neuronal $(\mathrm{NeuN}+)$ fractions. Thus, we cannot exclude the potential contributions of L1-mediated gene disruptions in the glial cells to the pathophysiology of SZ.

In summary, this study presents substantial evidence that PDS harbor L1 insertions in genes implicated in the pathophysiology of SZ. The convergence of genes identified here with those of other studies of SZ (GWAS, CNV, and so on), and consistencies between our results and those of Bundo et al (2014) lend credence to these results. However, these data require extension to independent groups of postmortem DLPFC samples from PDS. Whether novel L1 insertions are the cause or the result of putative alterations in brain development in PDS remains undetermined. If L1-mediated gene disruption during neurodevelopment increases risk for SZ, then genes identified with such disruptions may present new opportunities for the development of novel therapeutic approaches to treat this devastating disease.

\section{FUNDING AND DISCLOSURE}

This work was supported by NIH/NIMH grants 1R21MH101065 and 1R01MH109260-01A1 to WHB, NIH/ NIMH p50 grant MH096891 to CGH, and NIH/NIDA grant K01-036751 to RCC. ADE was supported by an ARC Discovery Early Career Researcher Award DE150101117 and the Mater Foundation. The authors declare no conflict of interest.

\section{ACKNOWLEDGMENTS}

We thank Paul Hallberg for performing the fluorescenceactivated cell sorter separations at the University of 
Pennsylvania Flow Cytometry Core Facility. We also thank the Children's Hospital of Philadelphia Nucleic Acid/Protein core for performing the Sanger sequencing. We acknowledge the NIMH Center for Collaborative Genomic Studies on Mental Disorders established through the NIMH Human Genetics Initiative in conjunction with Washington University in Saint Louis, the University of Southern California's Information Sciences Institute, and Rutgers University's RUCDR Infinite Biologics for providing DNA samples collected from the following studies and investigators: subjects were obtained from the National Institute of Mental Health Schizophrenia Genetics Initiative (NIMH-GI); data and biomaterials were collected by the 'Molecular Genetics of Schizophrenia II' (MGS-2) collaboration. The investigators and coinvestigators are as follows: ENH/Northwestern University, Evanston, IL, MH059571, Pablo V Gejman, MD (Collaboration Coordinator, PI), and Alan R Sanders, MD; Emory University School of Medicine, Atlanta, GA, MH59587, Farooq Amin, MD (PI); Louisiana State University Health Sciences Center; New Orleans, LA, MH067257, Nancy Buccola, APRN, BC, MSN (PI); University of California-Irvine, Irvine, CA, MH60870, William Byerley, MD (PI); Washington University, St. Louis, MO, U01, MH060879, C Robert Cloninger, MD (PI); University of Iowa, Iowa City, IA, MH59566, Raymond Crowe, MD (PI) and Donald Black, MD; University of Colorado, Denver, CO, MH059565, Robert Freedman, MD (PI); University of Pennsylvania, Philadelphia, PA, MH061675, Douglas Levinson MD (PI); University of Queensland, Queensland, Australia, MH059588, Bryan Mowry, MD (PI); and Mt. Sinai School of Medicine, New York, NY, MH59586, Jeremy Silverman, $\mathrm{PhD}$ (PI). The samples were collected by Vishwajit Nimgaonkar's group at the University of Pittsburgh, as part of a multi-institutional collaborative research project with Jordan Smoller, MD, DSc, and Pamela Sklar, MD, PhD, Massachusetts General Hospital (grant MH63420). Data and biomaterials generated in Study 6 were collected by the Molecular Genetics of Schizophrenia, part 1 (MGS1), and funded by collaborative NIMH grants to Evanston Northwestern Healthcare/Northwestern University, Evanston, IL, MH059571, Pablo V Gejman, MD (Collaboration Coordinator; PI), and Alan R Sanders, MD; Emory University School of Medicine, Atlanta, GA, MH59587, Farooq Amin, MD (PI); University of California, San Francisco, CA, MH60870, William Byerley, MD (PI); University of Iowa, Iowa City, IA, MH59566, Raymond Crowe, MD (PI), Donald Black, MD; Washington University, St. Louis, MO, U01, MH060879, C Robert Cloninger, MD (PI); University of Colorado, Denver, CO, MH059565, Robert Freedman, MD (PI), Ann Olincy, MD; University of Pennsylvania, Philadelphia, PA, MH061675, Douglas Levinson, MD (PI), Nancy Buccola, APRN, BC, MSN, New Orleans, LA; University of Queensland, Queensland, Australia, MH059588, Bryan Mowry, MD (PI); and Mt. Sinai School of Medicine, New York, NY, MH059586, Jeremy Silverman, PhD (PI). Data and biomaterials used in Study 23 were collected by the University of Pittsburgh and funded by an NIMH grant (Genetic Susceptibility in Schizophrenia, MH 56242) to Vishwajit Nimgaonkar, MD, PhD. Additional Principal Investigators on this grant include Smita Deshpande, MD, and Dr Ram Moanohar Lohia Hospital, New Delhi, India; and Michael Owen, MD, PhD, University of
Wales College of Medicine, Cardiff, UK. Most importantly, we thank the families who have participated in and contributed to these studies.

\section{REFERENCES}

Arnold SE, Gur RE, Shapiro RM, Fisher KR, Moberg PJ, Gibney MR et al (1995). Prospective clinicopathologic studies of schizophrenia: accrual and assessment of patients. Am J Psychiatry 152: 731-737.

Beck CR, Collier P, Macfarlane C, Malig M, Kidd JM, Eichler EE et al (2010). LINE-1 retrotransposition activity in human genomes. Cell 141: 1159-1170.

Beck JA, Poulter M, Campbell TA, Uphill JB, Adamson G, Geddes JF et al (2004). Somatic and germline mosaicism in sporadic earlyonset alzheimer's disease. Hum Mol Genet 13: 1219-1224.

Brouha B, Schustak J, Badge RM, Lutz-Prigge S, Farley AH, Moran JV et al (2003). Hot L1s account for the bulk of retrotransposition in the human population. Proc Natl Acad Sci USA 100: 5280-5285.

Brown SM, Clapcote SJ, Millar JK, Torrance HS, Anderson SM, Walker R et al (2011). Synaptic modulators Nrxn1 and Nrxn3 are disregulated in a Disc1 mouse model of schizophrenia. Mol Psychiatry 16: 585-587 Erratum in: Mol Psychiatry, 2012 17:469.

Bundo M, Toyoshima M, Okada Y, Akamatsu W, Ueda J, Nemoto-Miyauchi T et al (2014). Increased L1 retrotransposition in the neuronal genome in schizophrenia. Neuron 81: 306-313.

Carter CS, Barch DM, Gur R, Pinkham A, Ochsner K (2009). CNTRICS final task selection: social cognitive and affective neuroscience-based measures. Schizophr Bull 35: 153-162.

Carter CS, Perlstein W, Ganguli R, Brar J, Mintun M, Cohen JD (1998). Functional hypofrontality and working memory dysfunction in schizophrenia. Am J Psychiatry 155: 1285-1287.

Coufal NG, Garcia-Perez JL, Peng GE, Yeo GW, Mu Y, Lovci MT et al (2009). L1 retrotransposition in human neural progenitor cells. Nature 460: 1127-1131.

Costas J, Suárez-Rama JJ, Carrera N, Paz E, Páramo M, Agra S et al (2013). Role of DISC1 interacting proteins in schizophrenia risk from genome-wide analysis of missense SNPs. Ann Hum Genet 77: $504-512$.

Curtis VA, Bullmore ET, Morris RG, Brammer MJ, Williams SC et al (1999). Attenuated frontal activation in schizophrenia may be task dependent. Schizophr Res 37: 35-44.

de Boer M, van Leeuwen K, Geissler J, Weemaes CM, van den Berg TK, Kuijpers TW et al (2014). Primary immunodeficiency caused by an exonized retroposed gene copy inserted in the CYBB gene. Hum Mutat 35: 486-496.

Duchaine TF, Wohlschlegel JA, Kennedy S, Bei Y, Conte D Jr, Pang $\mathrm{K}$ et al (2006). Functional proteomics reveals the biochemical niche of C. elegans DCR-1 in multiple small-RNAmediated pathways. Cell 124: 343-354.

Evrony GD, Cai X, Lee E, Hills LB, Elhosary PC, Lehmann HS et al (2012). Single-neuron sequencing analysis of L1 retrotransposition and somatic mutation in the human brain. Cell 151: 483-496.

Ewing AD, Kazazian HH Jr (2010). Whole-genome resequencing allows detection of many rare LINE-1 insertion alleles in humans. Genome Res 21: 985-990.

Feng Q, Moran JV, Kazazian HH Jr, Boeke JD (1996). Human L1 retrotransposon encodes a conserved endonuclease required for retrotransposition. Cell 87: 905-916.

Goodier JL (2014). Retrotransposition in tumors and brains. Mob DNA 5: 11.

Gur RC, Ragland JD, Moberg PJ, Biler WB, Kohler C, Siegel SJ et al (2001). Computerized neurocognitive scanning: the profile of Schizophrenia. Neuropsychopharmacology 25: 777-788.

Hancks DC, Kazazian HH Jr (2016). Roles for retrotransposon insertions in human disease. Mob DNA 7: 9. 
Huang DW, Sherman BT, Lempicki RA (2009). Systematic and integrative analysis of large gene lists using DAVID bioinformatics resources. Nat Protoc 4: 44-57.

Iijima T, Iijima Y, Witte H, Scheiffele P (2014). Neuronal cell type-specific alternative splicing is regulated by the $\mathrm{KH}$ domain protein SLM1. J Cell Biol 204: 331-342.

Jiang Y, Matevossian A, Huang HS, Straubhaar J, Akbarian S (2008). Isolation of neuronal chromatin from brain tissue. BMC Neurosci 9: 42.

Kazazian HH Jr, Wong C, Youssoufian H, Scott AF, Phillips DG, Antonarakis SE (1988). Haemophilia A resulting from novel insertion of $\mathrm{L} 1$ sequences represents a novel mechanism for mutation in man. Nature 332: 164-166.

Kent WJ (2002). BLAT-the BLAST-like alignment tool. Genome Res 12: 656-664.

Lander ES, Linton LM, Birren B, Nusbaum C, Zody MC, Baldwin J et al (2001). Initial sequencing and analysis of the human genome. Nature 409: 860-921.

Langmead B, Salzberg SL (2012). Fast gapped-read alignment with Bowtie 2. Nat Methods 9: 357-359.

Lee KW, Woon PS, Teo YY, Sim K (2012). Genome wide association studies (GWAS) and copy number variation (CNV) studies of the major psychoses: what have we learnt? Neurosci Biobehav Rev 36: 556-571.

Li H, Handsaker B, Wysoker A, Fennell T, Ruan J, Homer N et al (2009). The Sequence Alignment/Map format and SAMtools. Bioinformatics 25: 2078-2079.

Lichtenstein P, Yip BH, Björk C, Pawitan Y, Cannon TD, Sullivan PF et al (2009). Common genetic determinants of schizophrenia and bipolar disorder in Swedish families: a population-based study. Lancet 373: 234-239.

Lotan A, Fenckova M, Bralten J, Alttoa A, Dixson L, Williams RW et al (2014). Neuroinformatic analyses of common and distinct genetic components associated with major neuropsychiatric disorders. Front Neurosci 8: 331.

Macia A, Widmann TJ, Heras SR, Ayllon V, Sanchez L, Benkaddour-Boumzaouad $M$ et al (2017). Engineered LINE-1 retrotransposition in non-dividing human neurons. Genome Res 27: $335-348$

Manoach DS, Gollub RL, Benson ES, Searl MM, Goff DC et al (2000). Schizophrenic subjects show aberrant fMRI activation of dorsolateral prefrontal cortex and basal ganglia during working memory performance. Biol Psychiatry 48: 99-109.

Mathias SL, Scott AF, Kazazian HH Jr, Boeke JD, Gabriel A (1991). Reverse transcriptase encoded by a human transposable element. Science 254: 1808-1810.

Muotri AR, Chu VT, Marchetto MC, Deng W, Moran JV, Gage FH (2005). Somatic mosaicism in neuronal precursor cells mediated by L1 retrotransposition. Nature 435: 903-910.

Muotri AR, Marchetto MC, Coufal NG, Oefner R, Yeo G, Nakashima $\mathrm{K}$ et al (2010). L1 retrotransposition in neurons is modulated by MeCP2. Nature 468: 443-446.

Nakashima M, Miyajima M, Sugano H, Iimura Y, Kato M, Tsurusaki $\mathrm{Y}$ et al (2014). The somatic GNAQ mutation c.548G >A (p.R183Q) is consistently found in Sturge-Weber syndrome. J Hum Genet 59: 691-699.
Ostertag EM, Kazazian HH Jr (2001). Biology of mammalian L1 retrotransposons. Annu Rev Genet 35: 501-538.

Ripke S, Neale BM, Corvin A, Walters JTR, Farh K-H, Holmans PA et al (2014). Biological Insights from 108 schizophreniaassociated genetic loci. Nature 511: 421-427.

Roos D, Kuhns DB, Maddalena A, Bustamante J, Kannengiesser C, de Boer $\mathrm{M}$ et al (2010). Hematologically important mutations: the autosomal recessive forms of chronic granulomatous disease. Blood Cells Mol Dis 44: 291-299.

Rozen S, Skaletsky H (2000). Primer3 on the WWW for general users and for biologist programmers. In: Krawetz S, Misener S (eds). Bioinformatics Methods and Protocols. Humana Press: Totowa, NJ. pp 365-386.

Shirley MD, Tang H, Gallione CJ, Baugher JD, Frelin LP, Cohen B et al (2013). Sturge-Weber syndrome and port-wine stains caused by somatic mutation in GNAQ. N Engl J Med 368: 1971.

Spielhaupter C, Schätzl HM (2001). PrPC directly interacts with proteins involved in signaling pathways. $J$ Biol Chem 276: 44604-44612.

Sudmant PH, Rausch T, Gardner EJ, Handsaker RE, Abyzov A, Huddleston $J$ et al (2015). An integrated map of structural variation in 2,504 human genomes. Nature 526: 75-81.

Swerdlow NR, Light GA, Trim RS, Breier MR, Hines SR, Powell SB (2013). Forebrain gene expression predicts deficits in sensorimotor gating after isolation rearing in male rats. Behav Brain Res 257: $118-128$.

Thivierge C, Makil N, Flamand M, Vasale JJ, Mello CC, Wohlschlegel J et al (2011). Tudor domain ERI-5 tethers an RNA-dependent RNA polymerase to DCR-1 to potentiate endo-RNAi. Nat Struct Mol Biol 19: 90-97. Erratum in: Nat Struct Mol Biol, 2013 20:244.

Verdurand M, Fillman SG, Weickert CS, Zavitsanou K (2013). Increases in $[3 \mathrm{H}]$ muscimol and $[3 \mathrm{H}]$ flumazenil binding in the dorsolateral prefrontal cortex in schizophrenia are linked to $\hat{\mathrm{I}} \pm 4$ and $\hat{I}^{3} 2$ S mRNA levels respectively. PLoS ONE 8: e52724.

Vogt J, Bengesser K, Claes KB, Wimmer K, Mautner VF, van Minkelen R et al (2007). SVA retrotransposon insertionassociated deletion represents a novel mutational mechanism underlying large genomic copy number changes with nonrecurrent breakpoints. Genome Biol 15: R80.

Weinberger DR, Berman KF, Zec RF (1986). Physiologic dysfunction of dorsolateral prefrontal cortex in schizophrenia. I. Regional cerebral blood flow evidence. Arch Gen Psychiatry 43: 114-124.

White TB, McCoy AM, Streva VA, Fenrich J, Deininger PL (2014). A droplet digital PCR detection method for rare L1 insertions in tumors. Mob DNA 5: 30.

Xu W, Cohen-Woods S, Chen Q, Noor A, Knight J, Hosang G et al (2014). Genome-wide association study of bipolar disorder in Canadian and UK populations corroborates disease loci including SYNE1 and CSMD1. BMC Med Genet 15: 2.

Yuen EY, Jiang Q, Chen P, Feng J, Yan Z (2008). Activation of 5-HT2A/C receptors counteracts 5-HT1A regulation of n-methyl$\mathrm{D}$-aspartate receptor channels in pyramidal neurons of prefrontal cortex. J Biol Chem 283: 17194-17204.

Supplementary Information accompanies the paper on the Neuropsychopharmacology website (http://www.nature.com/npp) 